\title{
Anosov theorem for coincidences on nilmanifolds
}

\author{
by \\ Seung Won Kim and Jong Bum Lee (Seoul)
}

\begin{abstract}
Suppose that $L, L^{\prime}$ are simply connected nilpotent Lie groups such that the groups $\gamma_{i}(L)$ and $\gamma_{i}\left(L^{\prime}\right)$ in their lower central series have the same dimension. We show that the Nielsen and Lefschetz coincidence numbers of maps $f, g: \Gamma \backslash L \rightarrow \Gamma^{\prime} \backslash L^{\prime}$ between nilmanifolds $\Gamma \backslash L$ and $\Gamma^{\prime} \backslash L^{\prime}$ can be computed algebraically as follows:

$$
L(f, g)=\operatorname{det}\left(G_{*}-F_{*}\right), \quad N(f, g)=|L(f, g)|,
$$

where $F_{*}, G_{*}$ are the matrices, with respect to any preferred bases on the uniform lattices $\Gamma$ and $\Gamma^{\prime}$, of the homomorphisms between the Lie algebras $\mathfrak{L}, \mathfrak{L}^{\prime}$ of $L, L^{\prime}$ induced by $f, g$.
\end{abstract}

1. Introduction. Let $M$ and $N$ be closed manifolds, and $f, g: M \rightarrow N$ continuous maps. Then we define

$$
\operatorname{Coin}(f, g)=\{x \in M \mid f(x)=g(x)\},
$$

the coincidence set of $f$ and $g$. Coincidence theory for pairs $f, g$ is a natural extension of fixed point theory for a self-map $f: M \rightarrow M$. There are well known invariants in coincidence theory which are the Lefschetz coincidence number $L(f, g)$ and Nielsen coincidence number $N(f, g)$.

Suppose that $M, N$ are closed orientable manifolds of the same dimension $n$. Then $L(f, g)$ is defined and $L(f, g) \neq 0$ implies the existence of a coincidence for any maps $f^{\prime}, g^{\prime}$ which are homotopic to $f, g$, respectively. The definition of $L(f, g)$ is in [13, Chap. 7]. The Nielsen coincidence number $N(f, g)$ is a non-negative integer with the property that any two maps $f^{\prime}, g^{\prime}$ which are homotopic to $f, g$, respectively, have at least $N(f, g)$ coincidences. In [12], Schirmer shows that if $n \geq 3$, then there are two maps $f^{\prime}, g^{\prime}$, homotopic to $f, g$ respectively, such that they have exactly $N(f, g)$ coincidences. Therefore, if $n \geq 3$ and $N(f, g)=0$, then there are coincidence free maps in the homotopy classes of $f, g$. Thus the Nielsen coincidence number is much

2000 Mathematics Subject Classification: 55M20, 54H25, 57S30.

Key words and phrases: nilmanifold, Lefschetz coincidence number, Nielsen coincidence number.

Supported in part by grant No. R14-2002-044-01002-0(2004) from ABRL of KOSEF. 
more powerful than the Lefschetz coincidence number but computing it is very hard.

In [2], Brooks, Brown, Pak and Taylor show that for a self-map $f: M \rightarrow M$ on a torus, the Nielsen number $N(f)$ and Lefschetz number $L(f)$ are equal up to sign, i.e.,

$$
N(f)=|L(f)|=\left|\operatorname{det}\left(I-f_{*}\right)\right|,
$$

where $f_{*}: \pi_{1}(M) \rightarrow \pi_{1}(M)$ is the homomorphism on $\pi_{1}(M)$ induced by $f$. In [1] and [4], this result is extended to compact nilmanifolds. Let $L$ be a connected, simply connected nilpotent Lie group, $\Gamma$ a uniform lattice of it, and $M=\Gamma \backslash L$ a nilmanifold. Any $f: M \rightarrow M$ is homotopic to a map obtained from an endomorphism $F: L \rightarrow L$ for which $F(\Gamma) \subset \Gamma$. Let $F_{*}$ be the corresponding endomorphism of the Lie algebra of $L$. Then $N(f)=|L(f)|=\left|\operatorname{det}\left(I-F_{*}\right)\right|$. In [10], McCord generalized this result to coincidences on nilmanifolds (see also [3], [5], [8] and [14]). If $M_{1}, M_{2}$ are nilmanifolds of the same dimension, then $N(f, g)=|L(f, g)|$ for any $f, g: M_{1} \rightarrow M_{2}$.

The purpose of this work is to offer an algebraic computation formula for the Nielsen and Lefschetz coincidence numbers of any pair of continuous maps between nilmanifolds $\Gamma \backslash L$ and $\Gamma^{\prime} \backslash L^{\prime}$. Suppose that $L, L^{\prime}$ are simply connected nilpotent Lie groups such that the groups $\gamma_{i}(L), \gamma_{i}\left(L^{\prime}\right)$ in the lower central series have the same dimension. Any continuous maps $f, g$ : $\Gamma \backslash L \rightarrow \Gamma^{\prime} \backslash L^{\prime}$ induce homomorphisms $\Phi_{*}, \Psi_{*}$ between the Lie algebras $\mathfrak{L}, \mathfrak{L}^{\prime}$. The uniform lattices $\Gamma$ and $\Gamma^{\prime}$ give rise to preferred bases for $\mathfrak{L}$ and $\mathfrak{L}^{\prime}$. Let $F_{*}, G_{*}$ be the matrices of the homomorphisms $\Phi_{*}, \Psi_{*}$ with respect to any preferred bases of $\Gamma, \Gamma^{\prime}$. Then we show that

$$
L(f, g)=\operatorname{det}\left(G_{*}-F_{*}\right), \quad N(f, g)=|L(f, g)| .
$$

Since every infra-nilmanifold admits a finite covering by a closed nilmanifold, the averaging formula for Nielsen coincidence numbers on infra-nilmanifolds in [9] will become a practical computation formula.

2. Anosov theorem for coincidences on nilmanifolds. Let $f, g$ : $\Gamma \backslash L \rightarrow \Gamma^{\prime} \backslash L^{\prime}$ be continuous maps between nilmanifolds $\Gamma \backslash L$ and $\Gamma^{\prime} \backslash L^{\prime}$ of the same dimension. In what follows, we shall fix liftings $\widetilde{f}, \widetilde{g}: L \rightarrow L^{\prime}$ of $f, g$. Then these liftings define homomorphisms $\varphi, \psi: \Gamma \rightarrow \Gamma^{\prime}$ as follows:

$$
\tilde{f} \gamma=\varphi(\gamma) \widetilde{f}, \quad \widetilde{g} \gamma=\psi(\gamma) \widetilde{g} .
$$

By [6], the homomorphisms $\varphi, \psi: \Gamma \rightarrow \Gamma^{\prime}$ extend uniquely to Lie group homomorphisms $\Phi, \Psi: L \rightarrow L^{\prime}$. Then they induce Lie algebra homomorphisms $\Phi_{*}, \Psi_{*}: \mathfrak{L} \rightarrow \mathfrak{L}^{\prime}$. Since $\Phi(\Gamma) \subset \Gamma^{\prime}$ and $\Psi(\Gamma) \subset \Gamma^{\prime}$, the endomorphisms $\Phi, \Psi$ induce maps $\varphi_{\#}, \psi_{\#}: \Gamma \backslash L \rightarrow \Gamma^{\prime} \backslash L^{\prime}$. Furthermore, $\varphi_{\#}$ and $f$ induce exactly the same homomorphism $\varphi: \Gamma \rightarrow \Gamma^{\prime}$, and $\psi_{\#}$ and $g$ induce exactly the same 
homomorphism $\psi: \Gamma \rightarrow \Gamma^{\prime}$. Since $\Gamma \backslash L$ and $\Gamma^{\prime} \backslash L^{\prime}$ are $K(\pi, 1)$-manifolds, $\varphi_{\#}$ and $f$ are homotopic and $\psi_{\#}$ and $g$ are homotopic. Since the Nielsen and Lefschetz coincidence numbers are homotopy invariants, we may assume in what follows that $f, g$ are induced by homomorphisms $\Phi, \Psi$ between the universal covering nilpotent Lie groups $L$ and $L^{\prime}$.

The homomorphisms $\varphi, \psi: \Gamma \rightarrow \Gamma^{\prime}$ define the Reidemeister action of $\Gamma$ on $\Gamma^{\prime}$ as follows:

$$
\Gamma \times \Gamma^{\prime} \rightarrow \Gamma^{\prime}, \quad\left(\gamma, \gamma^{\prime}\right) \mapsto \psi(\gamma) \gamma^{\prime} \varphi(\gamma)^{-1} .
$$

Denote the set of Reidemeister classes of $\Gamma^{\prime}$ determined by $f, g$ by $\mathcal{R}[f, g]$. Then the coincidence set Coin $(f, g)$ splits into a disjoint union of coincidence classes

$$
\operatorname{Coin}(f, g)=\coprod_{\left[\gamma^{\prime}\right] \in \mathcal{R}[f, g]} p\left(\operatorname{Coin}\left(\gamma^{\prime} \Phi, \Psi\right)\right) .
$$

Let $\Gamma$ be a uniform lattice of a connected, simply connected nilpotent Lie group $L$. Then $\Gamma$ is a finitely generated torsion-free nilpotent group. Recall that the lower central series of $\Gamma$ is defined inductively via $\gamma_{1}(\Gamma)=\Gamma$ and $\gamma_{i+1}(\Gamma)=\left[\gamma_{i}(\Gamma), \Gamma\right]$. Suppose that $\Gamma$ is c-step nilpotent, i.e., $\gamma_{c}(\Gamma) \neq 1$, but $\gamma_{c+1}(\Gamma)=1$. The isolator of a subgroup $H$ of $\Gamma$, denoted by $\sqrt[\Gamma]{H}$, is the set $\left\{x \in \Gamma \mid x^{k} \in H\right.$ for some $\left.k\right\}$. It is well known ([11, p. 473]) that the sequence

$$
\Gamma=\Gamma_{1}=\sqrt[\Gamma]{\gamma_{1}(\Gamma)} \supset \Gamma_{2}=\sqrt[\Gamma]{\gamma_{2}(\Gamma)} \supset \cdots \supset \Gamma_{c}=\sqrt[\Gamma]{\gamma_{c}(\Gamma)} \supset \Gamma_{c+1}=1
$$

forms a central series with $\Gamma_{i} / \Gamma_{i+1} \cong \mathbb{Z}^{k_{i}}$. Since $\sqrt[\Gamma]{\gamma_{i}(\Gamma)}=\Gamma \cap \gamma_{i}(L)$, $\sqrt[\Gamma]{\gamma_{i}(\Gamma)}$ is a uniform lattice of $\gamma_{i}(L)$ and hence the nilmanifolds $\sqrt[\Gamma]{\gamma_{i}(\Gamma)} \backslash \gamma_{i}(L)$ are naturally sitting inside the nilmanifold $\Gamma \backslash L$. Now we fix the orientations of all manifolds arising in the natural embeddings $\sqrt[\Gamma]{\gamma_{i}(\Gamma)} \backslash \gamma_{i}(L) \hookrightarrow \Gamma \backslash L$. This means that the fixed orientation of $\Gamma \backslash L$ induces the fixed orientations of all the submanifolds $\sqrt[\Gamma]{\gamma_{i}(\Gamma)} \backslash \gamma_{i}(L)$. We can choose a generating set

$$
\mathbf{a}=\left\{\mathbf{a}_{1}, \ldots, \mathbf{a}_{c}\right\}
$$

in such a way that $\Gamma_{i}$ is the group generated by $\mathbf{a}_{i}=\left\{a_{i 1}, \ldots, a_{i n_{i}}\right\}$ and $\Gamma_{i+1}$, and $\left\{\mathbf{a}_{i}, \ldots, \mathbf{a}_{c}\right\}$ determines the fixed orientation of $\sqrt[\Gamma]{\gamma_{i}(\Gamma)} \backslash \gamma_{i}(L)$ for each $i=1, \ldots, c$. We refer to $\mathbf{a}=\left\{\mathbf{a}_{1}, \ldots, \mathbf{a}_{c}\right\}$ as a preferred basis of $\Gamma$.

We use $\mathfrak{L}$ to indicate the Lie algebra of $L$. This Lie algebra $\mathfrak{L}$ has the same dimension and nilpotency class as $L$. Moreover, in the case of connected, simply connected nilpotent Lie groups it is known that the exponential map $\exp : \mathfrak{L} \rightarrow L$ is a diffeomorphism, We denote its inverse by log. If $L^{\prime}$ is another connected, simply connected nilpotent Lie group, with Lie algebra $\mathfrak{L}^{\prime}$, then we have the following properties: 
- For any homomorphism $\phi: L \rightarrow L^{\prime}$ of Lie groups, there exists a unique homomorphism $d \phi: \mathfrak{L} \rightarrow \mathfrak{L}^{\prime}$ (differential of $\phi$ ) of Lie algebras, making the following diagram commuting:

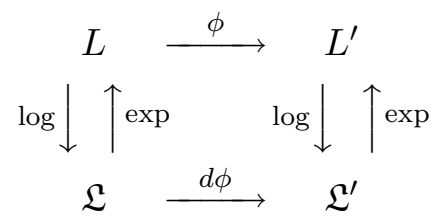

- Conversely, for any homomorphism $d \phi: \mathfrak{L} \rightarrow \mathfrak{L}^{\prime}$ of Lie algebras, there exists a unique homomorphism $\phi: L \rightarrow L^{\prime}$ of Lie groups, making the above diagram commuting.

If $\mathbf{a}$ is a preferred basis of $\Gamma$, then $\log \mathbf{a}=\left\{\log \mathbf{a}_{1}, \ldots, \log \mathbf{a}_{c}\right\} \subset \mathfrak{L}$ can be regarded as a basis for the vector space $\mathfrak{L}$. We also call it preferred. In particular, if $\Gamma$ is a uniform lattice of $\mathbb{R}^{d}$ then every preferred basis a of $\Gamma$ becomes a preferred basis $\log \mathbf{a}=\mathbf{a}$ for the vector space $\mathbb{R}^{d}$.

Lemma 2.1. Let $M=\Gamma \backslash L$ be a nilmanifold of dimension $d$ and $T=$ $\Gamma^{\prime} \backslash \mathbb{R}^{d}$ be a torus. Then for any continuous maps $f, g: M \rightarrow T$, we have

$$
L(f, g)=\operatorname{det}\left(G_{*}-F_{*}\right), \quad N(f, g)=|L(f, g)|,
$$

where $F_{*}, G_{*}$ are the $d \times d$ matrices, with respect to any preferred bases $\log \mathbf{a}$ and $\log \mathbf{a}^{\prime}$ of $\Gamma$ and $\Gamma^{\prime}$, of the homomorphisms from $\mathfrak{L}$ to $\mathbb{R}^{d}$ induced by $f, g: M \rightarrow T$.

Proof. If we assume that $M$ is also a torus then the result is known. Otherwise, the homomorphism $\Psi-\Phi: L \rightarrow \mathbb{R}^{d}$ from the non-abelian Lie group $L$ into the abelian Lie group $\mathbb{R}^{d}$ must be singular. In this case, $L(f, g)=N(f, g)=\operatorname{det}\left(G_{*}-F_{*}\right)=0$.

REMARK 2.2. Our original proof was longer, and this one was suggested by the referee.

Notation. For the commuting diagram

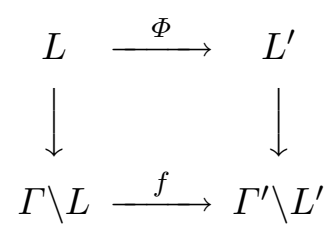

we shall use the following notations.

- $F_{*}$ is the matrix of the homomorphism $\Phi_{*}: \mathfrak{L} \rightarrow \mathfrak{L}^{\prime}$ with respect to any preferred bases $\log \mathbf{a}, \log \mathbf{a}^{\prime}$ of the uniform lattices $\Gamma, \Gamma^{\prime}$ respectively. That is,

$$
F_{*}=\left[\Phi_{*}\right]_{\log \mathbf{a}}^{\log \mathbf{a}^{\prime}} .
$$


- If $L=L^{\prime}$, then $f_{*}$ is the matrix of the homomorphism $\Phi_{*}: \mathfrak{L} \rightarrow \mathfrak{L}$ with respect to an arbitrarily chosen basis $\mathfrak{b}$ for $\mathfrak{L}$. That is,

$$
f_{*}=\left[\Phi_{*}\right]_{\mathfrak{b}}^{\mathfrak{b}} .
$$

EXAmPle 2.3. For any $c \in \mathbb{R}-\{0\}$, consider $f, g: \mathbb{Z} \backslash \mathbb{R} \rightarrow c \mathbb{Z} \backslash \mathbb{R}$. Then we have a commuting diagram

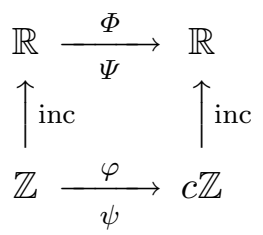

Here for some $a, b \in \mathbb{Z}, \Phi(x)=a(c x)$ and $\Psi(x)=b(c x)(x \in \mathbb{R})$. A preferred basis of $\mathbb{Z}$ is $\mathbf{a}=\{1\}$ and a preferred basis of $c \mathbb{Z}$ is $\mathbf{a}^{\prime}=\{c\}$. Thus $F_{*}=$ $\left[\Phi_{*}\right]_{\mathbf{a}}^{\mathbf{a}^{\prime}}=[a], G_{*}=\left[\Psi_{*}\right]_{\mathbf{a}}^{\mathbf{a}^{\prime}}=[b], f_{*}=\left[\Phi_{*}\right]_{\mathbf{a}}^{\mathbf{a}}=\left[\Phi_{*}\right]_{\mathbf{a}^{\prime}}^{\mathbf{a}^{\prime}}=[a c], g_{*}=[b c]$ and

$$
L(f, g)=\operatorname{det}\left(G_{*}-F_{*}\right)=b-a, \quad N(f, g)=|b-a| .
$$

The following is our main result.

Theorem 2.4. Let $M=\Gamma \backslash L$ and $M^{\prime}=\Gamma^{\prime} \backslash L^{\prime}$ be nilmanifolds. Suppose that the groups $\gamma_{i}(L)$ and $\gamma_{i}\left(L^{\prime}\right)$ in the lower central series of $L$ and $L^{\prime}$ have the same dimension. Then for any continuous maps $f, g: M \rightarrow M^{\prime}$, we have

$$
L(f, g)=\operatorname{det}\left(G_{*}-F_{*}\right), \quad N(f, g)=|L(f, g)| .
$$

Proof. Suppose that $L$ is a simply connected $c$-step nilpotent Lie group. Then $\gamma_{c}(L) \neq 1$ but $\gamma_{c+1}(L)=1$. Let $L_{c}=\gamma_{c}(L), \Gamma_{c}=\Gamma \cap L_{c}, L_{c}^{\prime}=\gamma_{c}\left(L^{\prime}\right)$ and $\Gamma_{c}^{\prime}=\Gamma^{\prime} \cap L_{c}^{\prime}$. Note that $\Gamma_{c}=\Gamma \cap \gamma_{c}(L)=\sqrt[\Gamma]{\gamma_{c}(\Gamma)}$ and $\Gamma_{c}^{\prime}=\sqrt[\Gamma^{\prime}]{\gamma_{c}\left(\Gamma^{\prime}\right)}$. Now we obtain principal fiber bundles $T \rightarrow M \rightarrow B$ and $T^{\prime} \rightarrow M^{\prime} \rightarrow B^{\prime}$, where $T=\Gamma_{c} \backslash L_{c}$ and $T^{\prime}=\Gamma_{c}^{\prime} \backslash L_{c}^{\prime}$ are tori of the same dimension, and $B=\left(\Gamma / \Gamma_{c}\right) \backslash\left(L / L_{c}\right)$ and $B^{\prime}=\left(\Gamma^{\prime} / \Gamma_{c}^{\prime}\right) \backslash\left(L^{\prime} / L_{c}^{\prime}\right)$ are nilmanifolds of the same dimension.

We may assume that the diagram

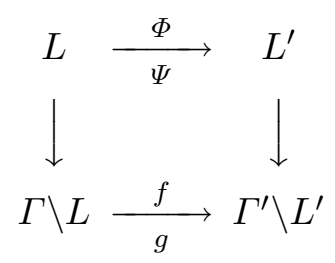

is commuting. The restrictions of $\Phi, \Psi: L \rightarrow L^{\prime}$ induce endomorphisms $\widehat{\Phi}, \widehat{\Psi}: L_{c} \rightarrow L_{c}^{\prime}$ and hence, in turn, endomorphisms $\bar{\Phi}, \bar{\Psi}: L / L_{c} \rightarrow L^{\prime} / L_{c}^{\prime}$ so that the following diagrams are commuting: 


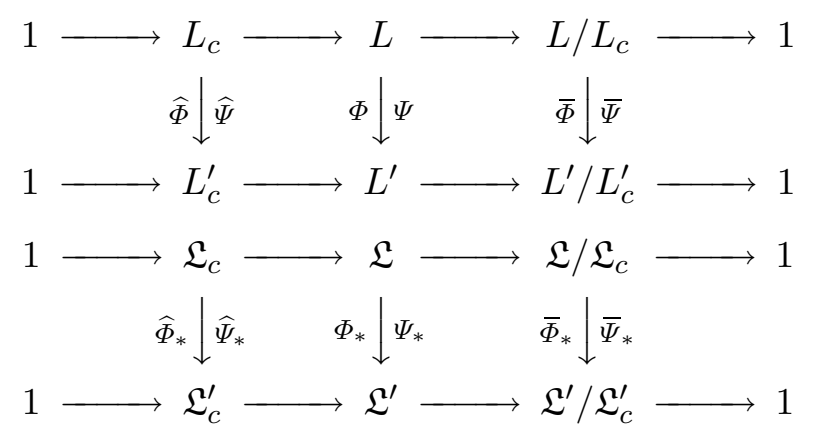

where $\mathfrak{L}_{c}$ is the Lie algebra of $L_{c}$ and so on. We choose any preferred basis $\log \mathbf{a}=\{\log \widehat{\mathbf{a}}, \log \overline{\mathbf{a}}\}$ of $\mathfrak{L}$ so that $\log \widehat{\mathbf{a}}$ is a preferred basis for $\mathfrak{L}_{c}$ and the image of $\log \overline{\mathbf{a}}$ in $\mathfrak{L} / \mathfrak{L}_{c}$ is a preferred basis for $\mathfrak{L} / \mathfrak{L}_{c}$. Similarly we choose any preferred basis $\log \mathbf{a}^{\prime}=\left\{\log \widehat{\mathbf{a}}^{\prime}, \log \overline{\mathbf{a}}^{\prime}\right\}$ of $\mathfrak{L}^{\prime}$.

Then $\Phi_{*}$ and $\Psi_{*}$ have matrices of the form

$$
F_{*}=\left[\begin{array}{cc}
\widehat{F}_{*} & * \\
0 & \bar{F}_{*}
\end{array}\right], \quad G_{*}=\left[\begin{array}{cc}
\widehat{G}_{*} & * \\
0 & \bar{G}_{*}
\end{array}\right],
$$

where $\widehat{F}_{*}, \widehat{G}_{*}, \bar{F}_{*}$ and $\bar{G}_{*}$ are the matrices with respect to the preferred bases $\log \widehat{\mathbf{a}}, \log \widehat{\mathbf{a}}^{\prime}$, the image of $\log \overline{\mathbf{a}}$ and the image of $\log \overline{\mathbf{a}}^{\prime}$.

Thus $\operatorname{det}\left(G_{*}-F_{*}\right)=\operatorname{det}\left(\widehat{G}_{*}-\widehat{F}_{*}\right) \cdot \operatorname{det}\left(\bar{G}_{*}-\bar{F}_{*}\right)$. Furthermore, $\widehat{\Phi}, \widehat{\Psi}$ map $\Gamma_{c}$ into $\Gamma_{c}^{\prime}$, and $\bar{\Phi}, \bar{\Psi}$ map $\Gamma / \Gamma_{c}$ into $\Gamma^{\prime} / \Gamma_{c}^{\prime}$. Thus they induce maps $\widehat{f}, \widehat{g}$ : $T \rightarrow T^{\prime}$ and $\bar{f}, \bar{g}: B \rightarrow B^{\prime}$ so that the following diagram is commutative:

$$
\begin{array}{ccc}
T & M & \longrightarrow \\
\widehat{f} \downarrow \widehat{g} & f \downarrow g & \bar{f} \downarrow \\
T^{\prime} \longrightarrow & M^{\prime} \longrightarrow B^{\prime}
\end{array}
$$

Now we prove the theorem using induction on the nilpotency of $L$. On the tori, by Lemma 2.1 we have

$$
L(\widehat{f}, \widehat{g})=\operatorname{det}\left(\widehat{G}_{*}-\widehat{F}_{*}\right), \quad N(\widehat{f}, \widehat{g})=|L(\widehat{f}, \widehat{g})|,
$$

where $\widehat{F}_{*}$ and $\widehat{G}_{*}$ are the matrices with respect to the preferred bases $\log \widehat{\mathbf{a}}$ and $\log \widehat{\mathbf{a}}^{\prime}$ of the vector spaces $\mathfrak{L}_{c}$ and $\mathfrak{L}_{c}^{\prime}$ corresponding to any preferred bases $\widehat{\mathbf{a}}$ and $\widehat{\mathbf{a}}^{\prime}$ of the uniform lattices $\Gamma_{c}$ and $\Gamma_{c}^{\prime}$, respectively. By the induction hypothesis, we have

$$
L(\bar{f}, \bar{g})=\operatorname{det}\left(\bar{G}_{*}-\bar{F}_{*}\right), \quad N(\bar{f}, \bar{g})=|L(\bar{f}, \bar{g})|,
$$

where $\bar{F}_{*}$ and $\bar{G}_{*}$ are the matrices with respect to the preferred bases $\log \overline{\mathbf{a}}$ and $\log \overline{\mathbf{a}}^{\prime}$ of the vector spaces $\mathfrak{L} / \mathfrak{L}_{c}$ and $\mathfrak{L}^{\prime} / \mathfrak{L}_{c}^{\prime}$ corresponding to any preferred bases $\overline{\mathbf{a}}$ and $\overline{\mathbf{a}}^{\prime}$ of the uniform lattices $\Gamma / \Gamma_{c}$ and $\Gamma^{\prime} / \Gamma_{c}^{\prime}$, respectively. (Here we abuse notation: $\overline{\mathbf{a}} \subset \Gamma$ and the image of $\overline{\mathbf{a}}$ in $\Gamma / \Gamma_{c}$ is the preferred basis $\overline{\mathbf{a}}$.) 
If $L(\bar{f}, \bar{g})=0$, then $N(\bar{f}, \bar{g})=0$ by the induction hypothesis, and hence $(\bar{f}, \bar{g})$ is homotopic to a coincidence free pair. This fact follows from the Wecken type theorem if $\operatorname{dim} B=\operatorname{dim} B^{\prime} \geq 3$ (see [12]). If $\operatorname{dim} B=\operatorname{dim} B^{\prime}$ $<3$, then they are $T^{1}$ or $T^{2}$ and hence this fact is easily deduced. Next, this homotopy may be lifted to give rise to a deformation of $(f, g)$ to a coincidence free pair. Thus $N(f, g)=L(f, g)=0$.

Now assume that $L(f, g) \neq 0$. Then the assumptions of [8, Theorem 6.5] are satisfied, because the second fundamental group of any nilmanifold vanishes and $\operatorname{Coin}(\bar{\Phi}, \bar{\Psi})=\{0\}$ by [10, Lemma 2.5]. Thus the product formula $N(f, g)=N(\widehat{f}, \widehat{g}) \cdot N(\bar{f}, \bar{g})$ holds. Since the fibration $T^{\prime} \rightarrow M^{\prime} \rightarrow B^{\prime}$ is orientable, the formula $L(f, g)=L(\widehat{f}, \widehat{g}) \cdot L(\bar{f}, \bar{g})$ also holds. Hence

$$
|L(f, g)|=|L(\widehat{f}, \widehat{g}) \cdot L(\bar{f}, \bar{g})|=N(\widehat{f}, \widehat{g}) \cdot N(\bar{f}, \bar{g})=N(f, g),
$$

and

$$
L(f, g)=L(\widehat{f}, \widehat{g}) \cdot L(\bar{f}, \bar{g})=\operatorname{det}\left(\widehat{G}_{*}-\widehat{F}_{*}\right) \cdot \operatorname{det}\left(\bar{G}_{*}-\bar{F}_{*}\right)=\operatorname{det}\left(G_{*}-F_{*}\right) .
$$

Finally, suppose that $\log \mathbf{b}=\{\log \widehat{\mathbf{b}}, \log \overline{\mathbf{b}}\}$ and $\log \mathbf{b}^{\prime}=\left\{\log \widehat{\mathbf{b}^{\prime}}, \log \overline{\mathbf{b}^{\prime}}\right\}$ are other preferred bases of $\mathfrak{L}$ and $\mathfrak{L}^{\prime}$, respectively. We notice that the transition matrices from one preferred basis to another one (both corresponding to the same uniform lattice) have determinant +1 since both preferred bases determine the same orientation. Namely, the transition matrices $[\mathrm{id}] \log \mathbf{b} \mathbf{a}$ and $[\mathrm{id}] \log \mathbf{a}^{\prime} \mathbf{b}^{\prime}$ have determinant +1 . Since

$$
\begin{aligned}
& {\left[\Phi_{*}\right]_{\log \mathbf{a}}^{\log \mathbf{a}^{\prime}}=[\mathrm{id}] \log \mathbf{b}^{\prime} \mathbf{b}^{\prime} \cdot\left[\Phi_{*}\right]_{\log \mathbf{b}}^{\log \mathbf{b}^{\prime}} \cdot[\mathrm{id}]_{\log \mathbf{a}}^{\log \mathbf{b}},} \\
& \left.\left[\Psi_{*}\right]_{\log \mathbf{a}}^{\log \mathbf{a}^{\prime}}=[\mathrm{id}]\right]_{\log \mathbf{b}^{\prime}}^{\log \mathbf{a}^{\prime}} \cdot\left[\Psi_{*}\right]_{\log \mathbf{b}}^{\log \mathbf{b}^{\prime}} \cdot[\mathrm{id}]_{\log \mathbf{a}}^{\log \mathbf{b}},
\end{aligned}
$$

it follows that $\operatorname{det}\left(G_{*}-F_{*}\right)$ does not depend on the choice of the pairs of preferred bases $\mathbf{a}, \mathbf{a}^{\prime}$ and $\mathbf{b}, \mathbf{b}^{\prime}$ of $\Gamma, \Gamma^{\prime}$. This finishes the proof.

3. Example. For $\mathbf{x}=\left\{x_{1}, \ldots, x_{p}\right\} \subset \Gamma, \mathbf{X}=\left\{X_{1}, \ldots, X_{p}\right\} \subset \mathfrak{L}$, and a $p \times p$ integral matrix $N=\left(n_{i j}\right)$, we use the following notations:

$$
\begin{aligned}
\mathbf{x}^{N} & =\left\{x_{1}^{n_{11}} x_{2}^{n_{12}} \cdots x_{p}^{n_{1 p}}, \ldots, x_{1}^{n_{p 1}} x_{2}^{n_{p 2}} \cdots x_{p}^{n_{p p}}\right\}, \\
N \mathbf{X} & =\left\{n_{11} X_{1}+\cdots+n_{1 p} X_{p}, \ldots, n_{p 1} X_{1}+\cdots+n_{p p} X_{p}\right\} .
\end{aligned}
$$

Recall that for a uniform lattice $\Gamma$ of a simply connected nilpotent Lie group $L$, we let $\Gamma_{i}=\sqrt[\Gamma]{\gamma_{i}(\Gamma)}$; then a generating set

$$
\mathbf{a}=\left\{\mathbf{a}_{1}, \ldots, \mathbf{a}_{c}\right\}
$$

is a preferred basis of $\Gamma$ if and only if $\Gamma_{i}$ is the group generated by $\mathbf{a}_{i}$ and $\Gamma_{i+1}$ for each $i=1, \ldots, c$.

LEMMA 3.1. Let $\Gamma$ and $\Lambda$ be uniform lattices of a simply connected nilpotent Lie group L. Let $\mathbf{a}=\left\{\mathbf{a}_{1}, \ldots, \mathbf{a}_{c}\right\}$ be a preferred basis of $\Gamma$. If $\Lambda \subset \Gamma$, 
then there exists an upper triangular block integral matrix

$$
N=\left[\begin{array}{cccc}
N_{11} & N_{12} & \ldots & N_{1 c} \\
0 & N_{22} & \ldots & N_{2 c} \\
\vdots & \vdots & \ddots & \vdots \\
0 & 0 & \ldots & N_{c c}
\end{array}\right]
$$

such that the diagonal blocks have positive determinant, $[\Gamma: \Lambda]=\operatorname{det}(N)$ and $\Lambda$ has a preferred basis

$$
\mathbf{a}^{N}=\left\{\mathbf{a}_{1}^{N_{11}} \mathbf{a}_{2}^{N_{12}} \cdots \mathbf{a}_{c}^{N_{1 c}} ; \mathbf{a}_{2}^{N_{22}} \mathbf{a}_{3}^{N_{23}} \cdots \mathbf{a}_{c}^{N_{2 c}} ; \ldots ; \mathbf{a}_{c}^{N_{c c}}\right\} .
$$

If $\mathbf{b}$ is any preferred basis of $\Lambda$, then there exists an upper triangular block integral matrix $N$ whose diagonal blocks have positive determinant, $[\Gamma: \Lambda]=$ $\operatorname{det}(N)$ and $\mathbf{b}=\mathbf{a}^{N}$.

Proof. Let $\Lambda_{i}=\Lambda \cap \Gamma_{i}=\Lambda \cap \sqrt[\Gamma]{\gamma_{i}(\Gamma)}$ for $i=1, \ldots, c$. Then

$$
\Lambda=\Lambda_{1} \supset \cdots \supset \Lambda_{c} \supset \Lambda_{c+1}=1
$$

is a central series of $\Lambda$ with $\Lambda_{i} / \Lambda_{i+1} \cong \mathbb{Z}^{k_{i}}$ for each $i=1, \ldots, c$. In fact, there is a natural injection $\Lambda_{i} / \Lambda_{i+1} \rightarrow \Gamma_{i} / \Gamma_{i+1}$.

Since $\Lambda_{c} \subset \Gamma_{c}=\left\langle\mathbf{a}_{c}\right\rangle \cong \mathbb{Z}^{k_{c}}$, there is an integral matrix $N_{c c}$ with positive determinant such that $\mathbf{a}_{c}^{N_{c c}}$ is a generating set of $\Lambda_{c}$. Obviously $\left[\Gamma_{c}: \Lambda_{c}\right]=\operatorname{det}\left(N_{c c}\right)$. Next we consider the following commuting diagram of homomorphisms:

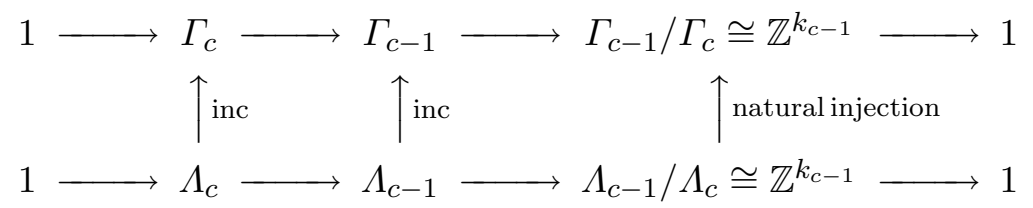

Since $\mathbf{a}_{c-1} \Gamma_{c}$ is a generating set of $\Gamma_{c-1} / \Gamma_{c}$, we can take $\mathbf{a}_{c-1}^{\prime} \subset \Lambda_{c-1}$ so that $\mathbf{a}_{c-1}^{\prime} \Lambda_{c}$ is a generating set of $\Lambda_{c-1} / \Lambda_{c}$. Then $\left\{\mathbf{a}_{c-1}^{\prime}, \mathbf{a}_{c}^{N_{c c}}\right\}$ is a generating set of $\Lambda_{c-1}$ and $\mathbf{a}_{c-1}^{\prime}=\mathbf{a}_{c-1}^{N_{c-1, c-1}} \mathbf{a}_{c}^{N_{c-1, c}}$, where $N_{c-1, c-1}$ and $N_{c-1, c}$ are integral matrices so that $\operatorname{det}\left(N_{c-1, c-1}\right)=\left[\Gamma_{c-1} / \Gamma_{c}: \Lambda_{c-1} / \Lambda_{c}\right]$. Moreover, $\left[\Gamma_{c-1}:\right.$ $\left.\Lambda_{c-1}\right]=\left[\Gamma_{c-1} / \Gamma_{c}: \Lambda_{c-1} / \Lambda_{c}\right] \cdot\left[\Gamma_{c}: \Lambda_{c}\right]=\operatorname{det}\left(N_{c-1, c-1}\right) \operatorname{det}\left(N_{c c}\right)$. Proceeding inductively, we obtain integral matrices $N_{11}, N_{12}, \ldots, N_{1 c} ; N_{22}, N_{23}, \ldots$, $N_{2 c} ; \ldots ; N_{c c}$ such that $\operatorname{det}\left(N_{i i}\right)$ are positive, $[\Gamma: \Lambda]=\operatorname{det}\left(N_{11}\right) \operatorname{det}\left(N_{22}\right) \ldots$ $\cdots \operatorname{det}\left(N_{c c}\right)$ and $\Lambda$ has a preferred basis

$$
\mathbf{a}^{N}=\left\{\mathbf{a}_{1}^{N_{11}} \mathbf{a}_{2}^{N_{12}} \cdots \mathbf{a}_{c}^{N_{1 c}} ; \mathbf{a}_{2}^{N_{22}} \mathbf{a}_{3}^{N_{23}} \cdots \mathbf{a}_{c}^{N_{2 c}} ; \ldots ; \mathbf{a}_{c}^{N_{c c}}\right\} .
$$

This proves the lemma.

Let $\Lambda \subset \Gamma$ be uniform lattices of a simply connected nilpotent Lie group $L$. Let $q: \Lambda \backslash L \rightarrow \Gamma \backslash L$ be the covering projection. Then we have 
the commuting diagrams

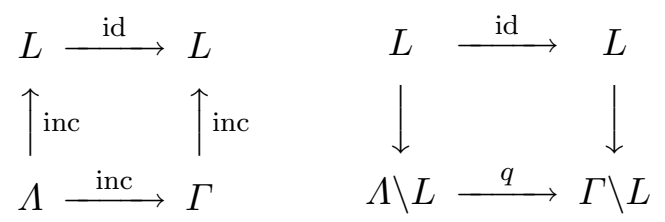

Let $\Gamma$ have a preferred basis $\mathbf{a}=\left\{\mathbf{a}_{1}, \ldots, \mathbf{a}_{c}\right\}$. By Lemma 3.1, $\Lambda$ has a preferred basis $\mathbf{b}=\mathbf{a}^{N}$ for some upper triangular block integral matrix $N$ with $[\Gamma: \Lambda]=\operatorname{det}(N)$.

To compare $\log \mathbf{b}$ with $\log \mathbf{a}$, we recall the famous Baker-CampbellHausdorff formula:

$$
\log (a \cdot b)=\log a * \log b \quad \text { for all } a, b \in L,
$$

where

$$
A * B=A+B+\frac{1}{2}[A, B]+\sum_{m=3}^{\infty} C_{m}(A, B) .
$$

Here $C_{m}(A, B)$ stands for a rational combination of $m$-fold Lie brackets in $A$ and $B$. Since our Lie algebra is nilpotent, the sum involved in $A * B$ is always finite.

Since $\mathbf{a}_{i} \subset \gamma_{i}(L)$, we have $\log \mathbf{a}_{i} \subset \log \gamma_{i}(L)=\gamma_{i}(\mathfrak{L})$. So, $\left[\log \mathbf{a}_{i}, \log \mathbf{a}_{i+1}\right]$ $\subset \gamma_{i+2}(\mathfrak{L})$. This implies that $\left[\log \mathbf{a}_{i}, \log \mathbf{a}_{i+1}\right]$ is a rational matrix linear combination of $\log \mathbf{a}_{j}$ where $j>i+1$. Thus

$$
\begin{aligned}
\log \left(\mathbf{a}_{i}^{N_{i}} \mathbf{a}_{i+1}^{N_{i+1}}\right. & \left.\cdots \mathbf{a}_{c}^{N_{c}}\right)=N_{i} \log \mathbf{a}_{i} \\
& + \text { a rational matrix linear combination of } \log \mathbf{a}_{i+1}, \ldots, \log \mathbf{a}_{c} .
\end{aligned}
$$

Therefore,

$$
\operatorname{det}\left(\left[q_{*}\right]_{\log \mathbf{b}}^{\log \mathbf{a}}\right)=\operatorname{det}\left([\mathrm{id}]_{\log \mathbf{b}}^{\log \mathbf{a}}\right)=\operatorname{det}\left[\begin{array}{cccc}
N_{11} & * & \ldots & * \\
0 & N_{22} & \ldots & * \\
\vdots & \vdots & \ddots & \vdots \\
0 & 0 & \ldots & N_{c c}
\end{array}\right]=[\Gamma: \Lambda] .
$$

We single this fact out as a lemma.

LEMMA 3.2. Let $\Lambda \subset \Gamma$ be uniform lattices of a simply connected nilpotent Lie group L. Let $q: \Lambda \backslash L \rightarrow \Gamma \backslash L$ be the covering projection. For any preferred bases $\mathbf{a}$ and $\mathbf{b}=\mathbf{a}^{N}$ of $\Gamma$ and $\Lambda$, respectively, we have

$$
\operatorname{det}\left(\left[q_{*}\right]_{\log \mathbf{b}}^{\log \mathbf{a}}\right)=\operatorname{det}\left([\mathrm{id}]_{\log \mathbf{b}}^{\log \mathbf{a}}\right)=[\Gamma: \Lambda] \text {. }
$$

The following is practically useful in computing the Nielsen and Lefschetz coincidence numbers on some nilmanifolds. 
Corollary 3.3. Let $\Gamma \backslash L$ and $\Gamma^{\prime} \backslash L$ be nilmanifolds, i.e., $\Gamma$ and $\Gamma^{\prime}$ are uniform lattices of the connected simply connected nilpotent Lie group $L$. Suppose $\Gamma \cap \Gamma^{\prime}$ is a uniform lattice of $L$. Then for any continuous maps $f, g: \Gamma \backslash L \rightarrow \Gamma^{\prime} \backslash L$, we have

$$
L(f, g)=\frac{\left[\Gamma^{\prime}: \Gamma \cap \Gamma^{\prime}\right]}{\left[\Gamma: \Gamma \cap \Gamma^{\prime}\right]} \operatorname{det}\left(g_{*}-f_{*}\right), \quad N(f, g)=|L(f, g)| .
$$

Proof. Let $\Lambda:=\Gamma \cap \Gamma^{\prime}$ be the uniform lattice of $L$. Thus $\Lambda$ has finite index in both $\Gamma$ and $\Gamma^{\prime}$. Choose preferred bases $\mathbf{b}, \mathbf{a}, \mathbf{a}^{\prime}$ of the uniform lattices $\Lambda, \Gamma, \Gamma^{\prime}$, respectively. Then by Lemma 3.1 we have $\mathbf{b}=\mathbf{a}^{N}=\mathbf{a}^{\prime N^{\prime}}$ for some upper triangular block integral matrices $N, N^{\prime}$ with $[\Gamma: \Lambda]=\operatorname{det}(N)$ and $\left[\Gamma^{\prime}: \Lambda\right]=\operatorname{det}\left(N^{\prime}\right)$. The endomorphisms $\Phi_{*}, \Psi_{*}$ induced by $f, g$ on the vector space $\mathfrak{L}$ with various preferred bases yield the commuting diagram

$$
\begin{array}{ccc}
(\mathfrak{L}, \log \mathbf{a}) & \stackrel{\Phi_{*}}{\longrightarrow} & \left(\mathfrak{L}, \log \mathbf{a}^{\prime}\right) \\
\Psi_{*} & \uparrow_{\text {id }} \\
(\mathfrak{L}, \log \mathbf{b}) \underset{\Phi_{*}}{\stackrel{\Psi_{*}}{\longrightarrow}}(\mathfrak{L}, \log \mathbf{b})
\end{array}
$$

The corresponding matrices thus satisfy

$$
\begin{aligned}
& \left.\left[\Phi_{*}\right]_{\log \mathbf{a}}^{\log \mathbf{a}^{\prime}} \cdot[\mathrm{id}]\right]_{\log \mathbf{b}}^{\log \mathbf{a}}=[\mathrm{id}] \log \mathbf{b} \mathbf{a}^{\prime} \cdot\left[\Phi_{*}\right]_{\log \mathbf{b}}^{\log \mathbf{b}} \\
& \left.\left[\Psi_{*}\right]_{\log \mathbf{a}}^{\log \mathbf{a}^{\prime}} \cdot[\mathrm{id}]\right]_{\log \mathbf{b}}^{\log \mathbf{a}}=[\mathrm{id}] \log \mathbf{b} \mathbf{b} \cdot\left[\Psi_{*}\right]_{\log \mathbf{b}}^{\log \mathbf{b}}
\end{aligned}
$$

or

$$
F_{*} \cdot[\mathrm{id}] \log \mathbf{b}=[\mathrm{id}] \log \mathbf{b} \mathbf{a}^{\prime} \cdot f_{*}, \quad G_{*} \cdot[\mathrm{id}]_{\log \mathbf{b}}^{\log \mathbf{a}}=[\mathrm{id}]_{\log \mathbf{b}}^{\log \mathbf{a}^{\prime}} \cdot g_{*}
$$

By Lemma 3.2,

$$
\left.\left.\operatorname{det}([\mathrm{id}]]_{\log \mathbf{b}}^{\log \mathbf{b}}\right)=[\Gamma: \Lambda], \quad \operatorname{det}([\mathrm{id}]]_{\log \mathbf{b}}^{\log \mathbf{a}^{\prime}}\right)=\left[\Gamma^{\prime}: \Lambda\right] .
$$

Theorem 2.4, together with the above observation, yields

$$
\begin{aligned}
L(f, g) & =\operatorname{det}\left(G_{*}-F_{*}\right) \\
& =\frac{\operatorname{det}([\mathrm{id}] \log \mathbf{b} \mathbf{b})}{\operatorname{det}([\mathrm{id}] \log \mathbf{a} \mathbf{b})} \operatorname{det}\left(g_{*}-f_{*}\right)=\frac{\left[\Gamma^{\prime}: \Gamma \cap \Gamma^{\prime}\right]}{\left[\Gamma: \Gamma \cap \Gamma^{\prime}\right]} \operatorname{det}\left(g_{*}-f_{*}\right), \\
N(f, g) & =|L(f, g)| .
\end{aligned}
$$

This finishes the proof.

EXAmPle 3.4. Let $L$ be the 3 -dimensional Heisenberg group. That is,

$$
L=\left\{\left[\begin{array}{ccc}
1 & x & z \\
0 & 1 & y \\
0 & 0 & 1
\end{array}\right] \mid x, y, z \in \mathbb{R}\right\} .
$$


We denote this general element by $\{x, y, z\}$. For any integer $k>0$, we consider the subgroups $\Gamma_{k}=\{\{m, n, l / k\} \mid m, n, l \in \mathbb{Z}\}$ of $L$. These are uniform lattices of $L$, and every uniform lattice of $L$ is isomorphic to some $\Gamma_{k}$. Let $\Phi: L \rightarrow L$ be an endomorphism. Then we have a commuting diagram

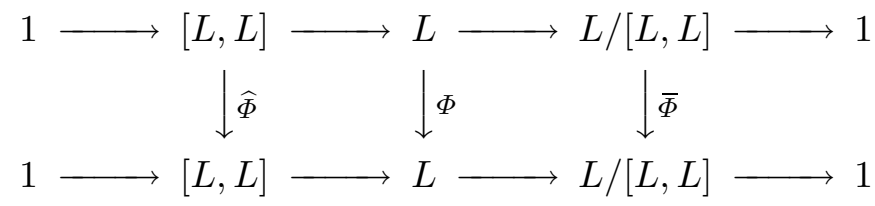

Since $[L, L]=\{\{0,0, z\} \mid z \in \mathbb{R}\}$ and $L /[L, L] \cong\{\{x, y, 0\} \mid x, y \in \mathbb{R}\}$, $\Phi$ must send $\{x, y, z\}$ to $\{\alpha x+\gamma y, \beta x+\delta y, \eta z+\varphi(x, y, z)\}$ for some $\alpha, \beta, \gamma, \delta, \eta$ $\in \mathbb{R}$. In particular, $\Phi(\{x, y, 0\} \cdot\{0,0, z\})=\Phi(\{x, y, 0\}) \cdot \Phi(\{0,0, z\})$ implies that $\varphi(x, y, z)=\varphi(x, y, 0)$. Thus $\varphi: \mathbb{R}^{2} \rightarrow \mathbb{R}$ is a function depending only on $x$ and $y$. Comparing the images of $\{x, y, 0\}=\{0, y, 0\} \cdot\{x, 0,0\}$ and $\{x, y, x y\}=\{x, 0,0\} \cdot\{0, y, 0\}$ under $\Phi$ shows that $\alpha \delta-\beta \gamma=\eta$.

Suppose $\Phi$ maps $\Gamma_{k}$ into $\Gamma_{k^{\prime}}$. Then $\alpha, \beta, \gamma, \delta \in \mathbb{Z}$ and $\Phi(\{0,0,1 / k\})=$ $\{0,0, \eta / k\}=\left\{0,0, l / k^{\prime}\right\}$ for some $l \in \mathbb{Z}$. Thus, if $\left(k, k^{\prime}\right)=m$, i.e., $k=m s$, $k^{\prime}=m t$ and $(s, t)=1$, then $\eta$ is a multiple of $s$ and $l$ is a multiple of $t$.

Let $\Phi, \Psi: L \rightarrow L$ be the endomorphisms of $L$ given by

$$
\begin{aligned}
& \Phi(\{x, y, z\})=\left\{2 x-2 y, 2 x+y, 6 z+2 x^{2}-4 x y-y^{2}\right\}, \\
& \Psi(\{x, y, z\})=\left\{3 y, x+y,-3 z+3 x y+\frac{3}{2} y^{2}\right\} .
\end{aligned}
$$

Then $\Phi\left(\Gamma_{6}\right) \subset \Gamma_{4}$ and $\Psi\left(\Gamma_{6}\right) \subset \Gamma_{4}$. Thus the endomorphisms $\Phi, \Psi: L \rightarrow L$ induce $f, g: \Gamma_{6} \backslash L \rightarrow \Gamma_{4} \backslash L$ so that the following diagram commutes:

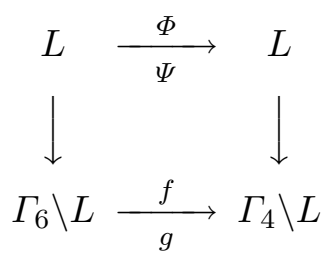

Since $\Gamma_{2}=\Gamma_{4} \cap \Gamma_{6}$, by Corollary 3.3 the Lefschetz and Nielsen coincidence numbers of $f, g$ are given by

$$
L(f, g)=\frac{2}{3} \operatorname{det}\left(g_{*}-f_{*}\right), \quad N(f, g)=|L(f, g)|,
$$

where $f_{*}, g_{*}$ are the matrices of the differentials of $\Phi, \Psi$ with respect to any basis of $\mathfrak{L}$.

We take an ordered (linear) basis for the Lie algebra $\mathfrak{L}$ of $L$ as follows:

$$
\mathbf{e}_{1}=\left[\begin{array}{lll}
0 & 0 & 1 \\
0 & 0 & 0 \\
0 & 0 & 0
\end{array}\right], \quad \mathbf{e}_{2}=\left[\begin{array}{lll}
0 & 1 & 0 \\
0 & 0 & 0 \\
0 & 0 & 0
\end{array}\right], \quad \mathbf{e}_{3}=\left[\begin{array}{lll}
0 & 0 & 0 \\
0 & 0 & 1 \\
0 & 0 & 0
\end{array}\right] \text {. }
$$


Note that this basis for $\mathfrak{L}$ is obtained from the preferred basis $\{0,0,1\}$, $\{1,0,0\},\{0,1,0\}$ for $\Gamma_{1}$. With respect to this basis, the differentials of $\Phi$ and $\Psi$ are

$$
f_{*}=\left[\begin{array}{rrr}
6 & 0 & 0 \\
0 & 2 & -2 \\
0 & 2 & 1
\end{array}\right], \quad g_{*}=\left[\begin{array}{rrr}
-3 & 0 & 0 \\
0 & 0 & 3 \\
0 & 1 & 1
\end{array}\right] .
$$

Therefore, the Lefschetz and Nielsen coincidence number of the maps $f, g$ : $\Gamma_{6} \backslash L \rightarrow \Gamma_{4} \backslash L$ are

$$
L(f, g)=\frac{2}{3} \operatorname{det}\left(g_{*}-f_{*}\right)=\frac{2}{3}(-45)=-30, \quad N(f, g)=30 .
$$

Acknowledgements. The authors would like to thank Professor J. Jezierski for pointing out some errors and valuable comments on the original version. They would also like to thank him for the information that similar computations were done in [7].

\section{References}

[1] D. V. Anosov, The Nielsen numbers of maps of nil-manifolds, Uspekhi Mat. Nauk 40 (1985), 133-134 (in Russian); English transl.: Russian Math. Surveys 40 (1985), $149-150$.

[2] R. B. S. Brooks, R. F. Brown, J. Pak and D. H. Taylor, Nielsen numbers of maps of tori, Proc. Amer. Math. Soc. 52 (1975), 398-400.

[3] R. B. S. Brooks and P. Wong, On changing fixed points and coincidences to roots, ibid. 115 (1992), 527-533.

[4] E. Fadell and S. Husseini, On a theorem of Anosov on Nielsen numbers for nilmanifolds, in: Nonlinear Functional Analysis and its Applications (Maratea, 1985), NATO Adv. Sci. Inst. Ser. C: Math. Phys. Sci. 173, Reidel, Dordrecht, 1986, 47-53.

[5] D. Gonçalves, The coincidence Reidemeister classes of maps on nilmanifolds, Topol. Methods Nonlinear Anal. 12 (1998), 375-386.

[6] V. V. Gorbachevich, Discrete subgroups of solvable Lie groups of type (E), Math. USSR-Sb. 40 (1971), 233-251.

[7] Ph. R. Heath and E. C. Keppelmann, Fibre techniques in Nielsen periodic point theory on solvmanifolds III. Calculations, Quaest. Math. 25 (2002), 177-208.

[8] J. Jezierski, The Nielsen number product formula for coincidences, Fund. Math. 134 (1989), 183-212.

[9] S. W. Kim and J. B. Lee, Averaging formula for Nielsen coincidence numbers, submitted, 2005.

[10] C. K. McCord, Lefschetz and Nielsen coincidence numbers on nilmanifolds and solvmanifolds, II, Topology Appl. 75 (1997), 81-92.

[11] D. S. Passman, The Algebraic Structure of Group Rings, Pure and Appl. Math., Wiley, New York, 1977.

[12] H. Schirmer, Mindestzahlen von Koinzidenzpunkten, J. Reine Angew. Math. 194 (1955), 21-39.

[13] J. Vick, Homology Theory: An Introduction to Algebraic Topology, 2nd ed., Grad. Texts in Math. 145, Springer, New York, 1994. 
[14] P. Wong, Reidemeister number, Hirsch rank, coincidences on polycyclic groups and solvmanifolds, J. Reine Angew. Math. 524 (2000), 185-204.

Department of Mathematics

Sogang University

Seoul 121-742, Korea

E-mail: swkim@sogang.ac.kr

jlee@sogang.ac.kr

Received 20 July 2004;

in revised form 10 May 2005 